\title{
A Case of Hypophosphatemia due to Oncogenic Osteomalacia in a Patient with Natural Killer T-Cell Lymphoma
}

\author{
Guoliang Zheng $^{\mathrm{a}}$ Swetha Rani Kandurib ${ }^{\mathrm{b}}$ John P. Canterbury ${ }^{\mathrm{b}}$ Thuy Nguyen ${ }^{\mathrm{a}}$ \\ Juan Carlos Q. Velez ${ }^{a}$, \\ aOchsner Clinical School/The University of Queensland, Brisbane, QLD, Australia; bepartment of Nephrology, \\ Ochsner Clinic Foundation, New Orleans, LA, USA
}

\section{Keywords}

Hypophosphatemia - Renal phosphate wasting - Oncogenic osteomalacia · Fibroblast growth factor-23 - Natural killer T-cell lymphoma $\cdot$ Tumor-induced osteomalacia

\begin{abstract}
Introduction: Oncogenic osteomalacia (Onc-Ost) is a paraneoplastic phenomenon characterized by hypophosphatemia due to elevated fibroblast growth factor-23 (FGF-23). Onc-Ost has been previously reported in patients with germ line mesenchymal tumors and solid organ malignancies. This is the first report of aggressive natural killer (NK) T-cell lymphoma presenting as Onc-Ost. Case Description: A 33-year-old Vietnamese female with active hepatitis $B$ and Mycobacterium avium complex, on ongoing therapy with tenofovir disoproxil, azithromycin, and ethambutol, presented with persistent fevers and developed refractory hypophosphatemia. Workup confirmed severe renal phosphate wasting. Tenofovir disoproxil was initially suspected; however, presence of isolated phosphaturia without Fanconi syndrome and persistence of hypophosphatemia despite discontinuation of medication led to clinical suspicion of Onc-
\end{abstract}

karger@karger.com www.karger.com/kbr

Karger"

BOPEN ACCESS
(C) 2021 The Author(s)

Published by S. Karger AG, Basel

This is an Open Access article licensed under the Creative Common Attribution-NonCommercial-4.0 International License (CC BY-NC) (http://www.karger.com/Services/OpenAccessLicense), applicable to the online version of the article only. Usage and distribution for commercial purposes requires written permission.
Ost. Elevated FGF-23 warranted further workup, leading to a definitive diagnosis of clinically subtle NK T-cell lymphoma. Chemotherapy was initiated; however, patient continued to deteriorate clinically and expired. Conclusion: Along with commonly reported germ line mesenchymal tumors and solid malignancies, NK T-cell lymphoma can also present as Onc-Ost. Timely detection of associated tumors and subsequent antitumor therapy would likely reverse hypophosphatemia and improve clinical outcomes.

(c) 2021 The Author(s)

Published by S. Karger AG, Basel

\section{Introduction}

Oncogenic osteomalacia (Onc-Ost), also known as tumor-induced osteomalacia, is a paraneoplastic syndrome characterized by constellation of elevated fibroblast growth factor-23 (FGF-23), hypophosphatemia, low to normal levels of $1,25-(\mathrm{OH})_{2}$ vitamin $\mathrm{D}$ (1,25 dihydroxy vitamin D), and phosphaturia [1]. Abnormal excessive production of FGF-23 by a tumor is recognized as the key element driving the disturbance in phosphate homeostasis. This syndrome is often associated with benign mes- 
enchymal tumors and is rarely reported among solid organ malignancies [2-5]. To our knowledge, the association of hypophosphatemia secondary to Onc-Ost in relation to natural killer (NK) T-cell lymphoma has not yet been reported. Herein, we report a case of 33-year-old Vietnamese female who presented with persistent fever, elevated inflammatory markers, and refractory hypophosphatemia secondary to Onc-Ost. Additionally, we discuss diagnostic and treatment strategies for patients with Onc-Ost.

\section{Case Description}

A 33-year-old Vietnamese female with active hepatitis B and Mycobacterium avium complex (MAC) infection, on ongoing therapy with tenofovir disoproxil, azithromycin, and ethambutol, presented to a regional medical center with recurrent fever, chills, productive cough, and dyspnea of 3-day duration. Upon arrival, the patient was tachycardic and tachypneic with diffuse rales on auscultation. Diagnosis of sepsis due to pneumonia was entertained, and the patient was started on fluids and broad-spectrum antibiotics. She eventually developed respiratory distress with hypoxemia requiring intubation and transfer to our academic medical center for further care. The patient was stabilized after admission to our intensive care unit. The patient developed bilateral pleural effusions that required thoracentesis on day 9 of hospital stay. Elevated ferritin and LDH and abundant lymphoid cells in pleural fluid led to preliminary diagnosis of hemophagocytic lymphohistiocytosis (HLH) that prompted initiation of dexamethasone and etoposide. It was noted that patient's phosphate levels were staying low around $2.3 \mathrm{mg} / \mathrm{dL}$ and dropped to as low as 1.3 $\mathrm{mg} / \mathrm{dL}$ despite intermittent supplementation, prompting nephrology consultation on day 20. Pertinent laboratory results obtained at time of consultation include serum creatinine of $0.5 \mathrm{mg} /$ $\mathrm{dL}$, corrected serum calcium at $8.5 \mathrm{mg} / \mathrm{dL}$, elevated alkaline phosphatase $701 \mathrm{U} / \mathrm{L}$ (55-135 U/L), parathyroid hormone (PTH) of $114 \mathrm{pg} / \mathrm{mL}(9-77 \mathrm{pg} / \mathrm{mL}), 1,25-(\mathrm{OH})_{2}$ vitamin D $20 \mathrm{pg} / \mathrm{mL}(20-$ $79 \mathrm{pg} / \mathrm{mL}$ ), random urinary phosphate of $63 \mathrm{mg} / \mathrm{dL}$, and fractional excretion of urinary phosphate of $43 \%$ (Table 1). Tenofovir disoproxil was initially considered as potential cause due to its proximal tubular toxicity and known association with Fanconi syndrome [6]. Additionally, tenofovir disoproxil has been reported to cause hyperparathyroidism by direct inhibition of calciumsensing receptors, as noted in our case [7]. However, isolated phosphaturia in the absence of glycosuria or aminoaciduria and persistent hypophosphatemia even after cessation of tenofovir prompted consideration for alternate diagnosis. Patient continued receiving aggressive oral and intravenous phosphate supplementation, and serum phosphate levels continued to fluctuate over the course of hospitalization (Fig. 1).

FGF-23 (C-Terminal) 2nd generation ELISA was ordered due to clinical suspicion of Onc-Ost. The FGF-23 levels returned significantly elevated at $1,940 \mathrm{RU} / \mathrm{mL}$ (normal: $<180 \mathrm{RU} / \mathrm{mL}$ ), supporting the diagnosis of Onc-Ost. At that point, given the complex clinical scenario of hepatitis B, MAC, HLH and Onc-Ost, a lymphoproliferative disorder was suspected. Bone marrow and lymph
Table 1. Laboratory values at the time of consultation to nephrology

\begin{tabular}{lrc}
\hline & $\begin{array}{c}\text { Patient } \\
\text { value }\end{array}$ & $\begin{array}{l}\text { Normal } \\
\text { range }\end{array}$ \\
\hline Serum & & \\
Sodium, mEq/L & 128 & $136-145$ \\
Potassium, mEq/L & 3.8 & $3.4-5.1$ \\
Bicarbonate, mEq/L & 24 & $22-29$ \\
Chloride, mEq/L & 105 & $96-105$ \\
Calcium, mg/dL & 5.9 & $8.6-10.2$ \\
Corrected calcium, mg/dL & 8.9 & $8.6-10.2$ \\
Albumin, g/dL & 1.5 & $3.8-4.5$ \\
Phosphate, mg/dL & 1.5 & $2.5-4.5$ \\
Creatinine, mg/dL & 0.5 & $0.5-1.1$ \\
Urea nitrogen, mg/dL & 15 & $6-23$ \\
Alkaline phosphatase, U/L & 701 & $35-104$ \\
PTH, pg/mL & 114 & $15-72$ \\
25-Hydroxyvitamin D, pg/mL & 8 & $30-100$ \\
1,25-Hydroxyvitamin D, pg/mL & 20 & $19.9-79.2$ \\
FGF-23, RU/mL & 1,940 & $<180$ \\
Urine & & \\
Phosphate, mg/dL & 63 & \\
Creatinine, mg/dL & 58 & \\
Fractional excretion of urinary phosphate, \% & 43 & \\
\hline
\end{tabular}

PTH, parathyroid hormone; FGF-23, fibroblast growth factor-23.

node biopsies were performed, and they revealed occasional EBV+ and CD5+ cells. A definitive diagnosis of NK T-cell lymphoma was established when flow cytometry from repeat thoracentesis performed on hospital day 30 revealed positive staining for CD2, CD7, and CD56 NK T cells. Patient was subsequently initiated on chemotherapy with dexamethasone, cisplatin, gemcitabine, and pegaspargase. She continued to be hypophosphatemic, needing phosphate supplementation. Patient continued to deteriorate clinically and expired on day 34 of hospitalization.

\section{Discussion}

Onc-Ost is characterized by persistent hypophosphatemia with clinical manifestations of fatigue, fractures, and other bone abnormalities. We report one of the first cases of Onc-Ost secondary to NK T-cell lymphoma. Our patient's complicated clinical presentation of ongoing infection with HBV, MAC, presumed diagnosis of HLH and possible tenofovir disoproxil-induced phosphate wasting made it particularly challenging to establish the final diagnosis. It was the elevated FGF-23 level, which increased the likelihood of Onc-Ost, prompting further investigations and ultimately leading to the final diagnosis of NK T-cell lymphoma. 


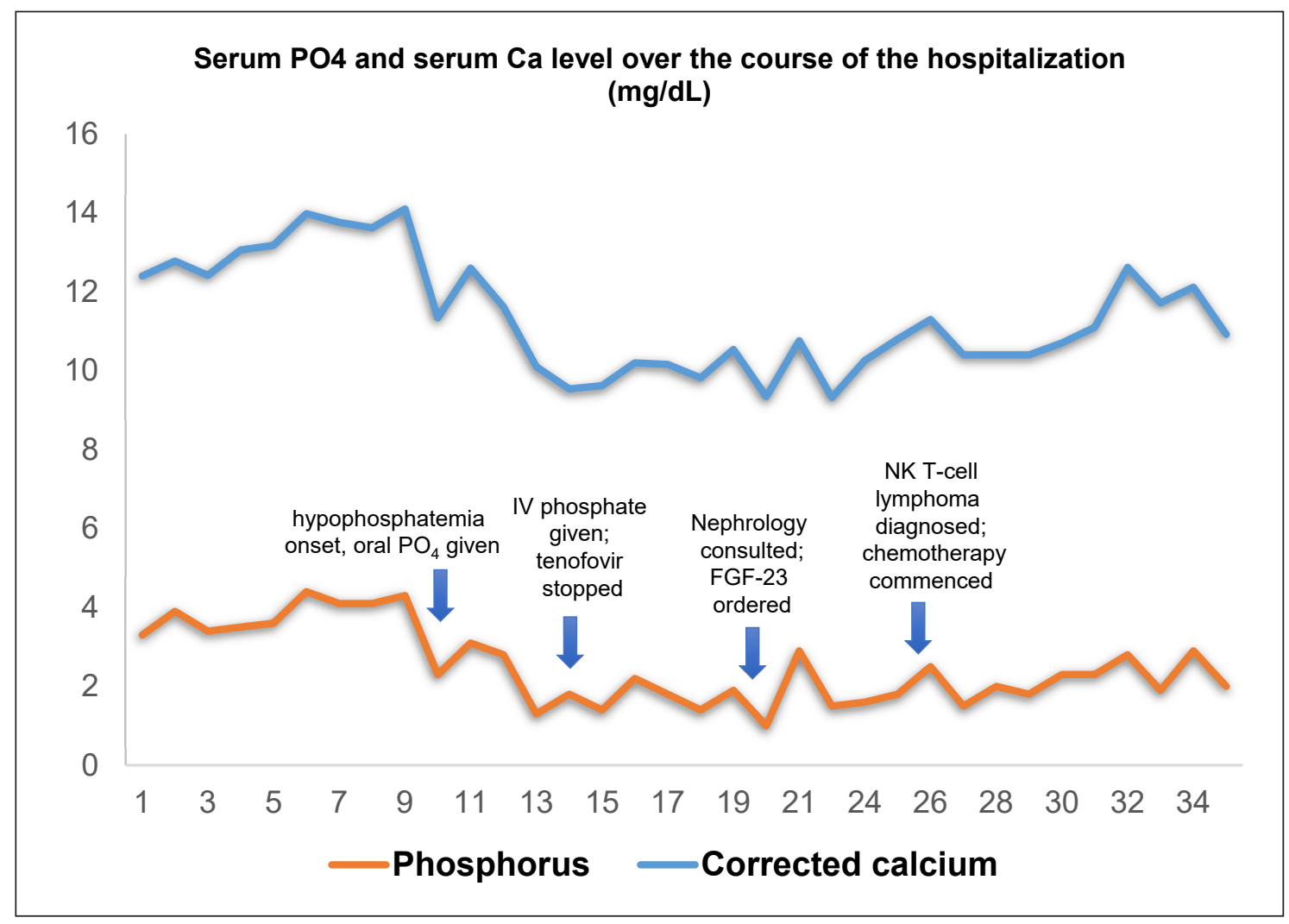

Fig. 1. Trajectory of serum phosphate and corrected serum calcium levels and timeline of major events over the course of the hospitalization. NK, natural killer; FGF-23, fibroblast growth factor-23; $\mathrm{PO}_{4}$, phosphate.

Tenofovir disoproxil in addition to multiple chemotherapeutic agents can cause proximal tubular damage, Fanconi syndrome, and concomitant phosphate wasting [6]. Even though hypophosphatemia secondary to phosphate wasting due to tenofovir disoproxil was initially entertained, lack of glucosuria, bicarbonaturia, lack of improvement of phosphate levels upon discontinuation of the drug, and elevated FGF-23 levels led to an accurate diagnosis. High FGF-23 levels further aided us in narrowing the differential of renal phosphate wasting disorders to Onc-Ost, X-linked hypophosphatemic rickets and autosomal dominant and recessive hypophosphatemic rickets, while the latter are mainly encountered in pediatric population [8].

In the index patient, persistent hypophosphatemia was associated with low active vitamin D and elevated alkaline phosphatase that are likely secondary to increased FGF23 peptides secreted by NK T-cell lymphoma. It is hypothesized that FGF-23 peptides secreted by tumors bind to fibroblast growth factor receptor 1 isoform IIIc found in proximal tubular epithelial cells and subsequently decrease the reabsorption of phosphate by inhibiting sodi- um-phosphate cotransporter (NaPi-IIa and IIc) [9]. In addition to directly affecting tubular handling of filtered phosphate, FGF-23 also inhibits 1-alpha hydroxylase in renal proximal tubule with subsequent decrease in 1,25$(\mathrm{OH})_{2}$ vitamin D levels, further increasing phosphate excretion. Additionally, FGF-23 demonstrates an inhibitory effect on PTH secretion in normocalcemic individuals [10]. However, elevated PTH levels can be seen as encountered in our case and are often driven by elevated FGF-23 and low vitamin D levels [11]. Figure 2 Illustrates pathogenesis of hypophosphatemia in Onc-Ost and role of FGF-23.

Onc-Ost is commonly reported among benign mesenchymal tumors such as nasal hemangiopericytoma [12] and femur mesenchymal tumor [13] and is rarely reported among other malignancies including small cell lung carcinoma [2], ovarian cancer [3], and metastatic breast cancer [4]. Elderman et al. [5] reported only case of Onc-Ost secondary to B-cell non-Hodgkin lymphoma, who presented with hypophosphatemia, renal phosphate wasting, and elevated FGF-23 levels. Unlike ours, patient in the report by Elderman et al. [5] 


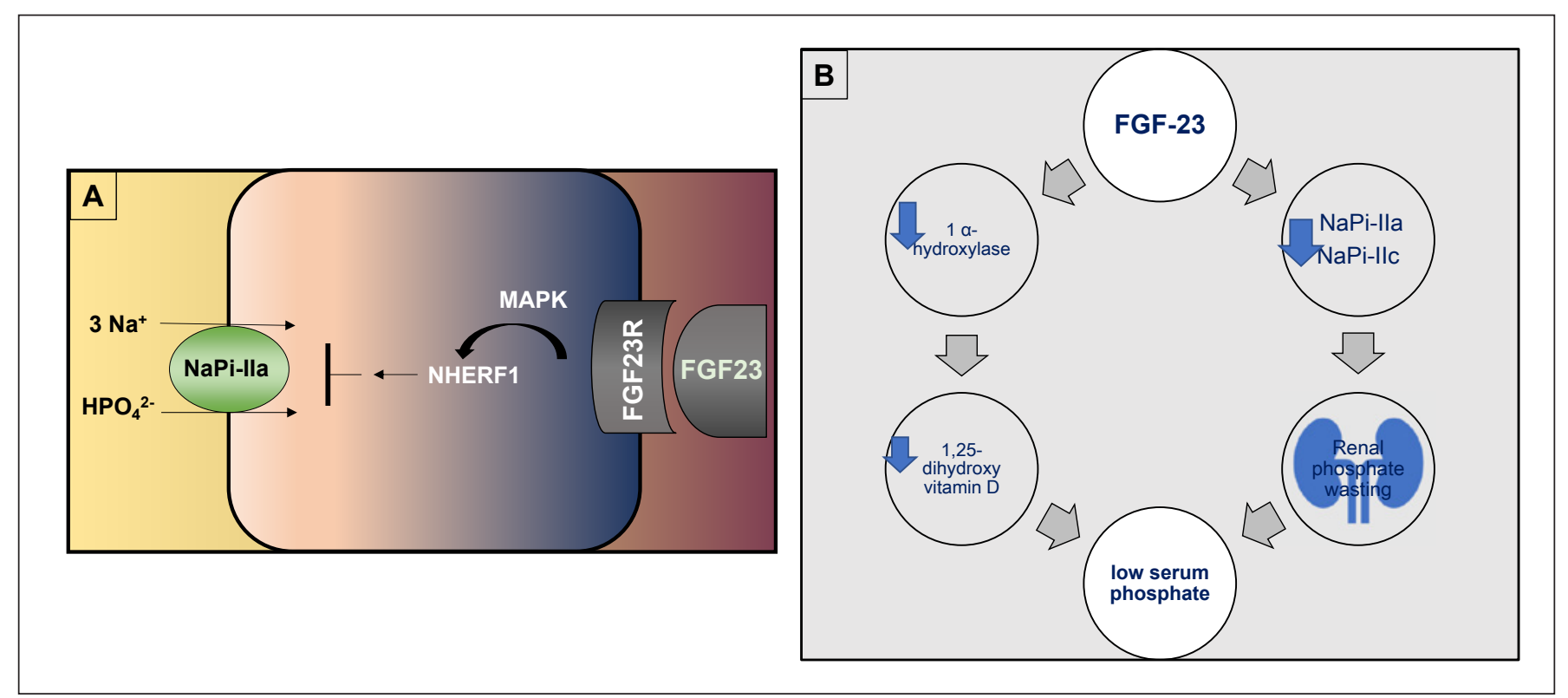

Fig. 2. Role of FGF-23 in the pathogenesis of Onc-Ost. a Effect of FGF-23 on inhibiting proximal tubular reabsorption of phosphate. $\mathbf{b}$ Schematic illustrating global effect of FGF-23 on vitamin D axis and intestinal phosphate handling as well as the pathway to alter direct tubular handling. Onc-Ost, oncogenic osteomalacia; FGF-23, fibroblast growth factor- 23 .

had a gingival lesion on physical examination and subsequent biopsy confirmed B-cell non-Hodgkin lymphoma. Prompt initiation of chemotherapy led to reversal of FGF-23 and serum phosphate levels within 2 weeks.

History and physical examination may provide vital information on localizing the tumor, but in cases where tumor size is too small, it is suggested to obtain functional imaging like octreotide scan [14], Gallium-68 DOTATATE PET/CT, and FDG-PET or anatomical imaging techniques including whole-body magnetic resonance imaging [15] or computed tomography [16]. Table 2 illustrates diagnostic tests to be considered in patient with renal phosphate wasting. Treatment of Onc-Ost is adequate antitumor therapy. When tumors are unresectable or with multiple recurrences, calcitriol and oral phosphate supplementation is recommended [17]. Human monoclonal antibody therapy against FGF-23, burosum$\mathrm{ab}$, initially approved for the treatment of X-linked hypophosphatemia [18], is currently been evaluated among patients with Onc-Ost. However, in our patient due to complicated clinical presentation, the diagnosis of NK T-cell lymphoma could not be made early enough and the patient expired within 4 days of chemotherapy initiation.
Table 2. Diagnostic tests to be considered in patient with renal phosphate wasting

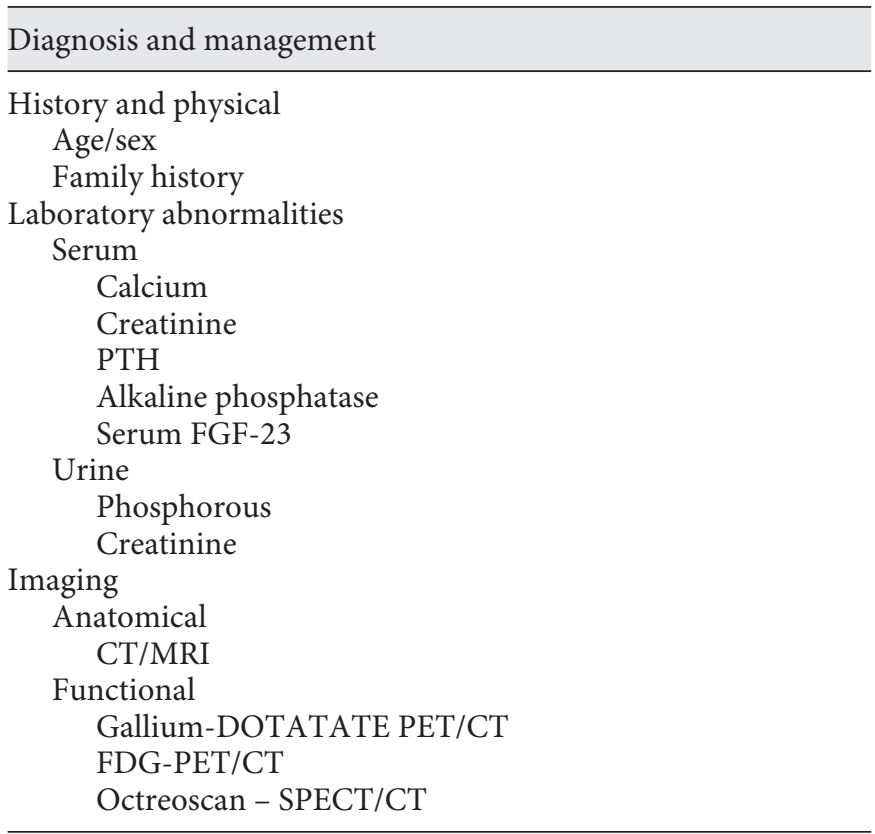

PTH, parathyroid hormone; FGF-23, fibroblast growth factor-23; PET/CT, positron emission tomography with computed tomography; FDG-PET/CT, F-18 fluorodeoxyglucose positron emission tomography with computed tomography; SPECT/CT, single photon emission and computed tomography. 


\section{Conclusion}

Onc-Ost should be considered in the workup of refractory hypophosphatemia secondary to renal phosphate wasting, and this could potentially lead to early discovery of clinically subtle malignancies. Obtaining FGF-23 level will likely play a key role in narrowing the differential diagnosis of renal phosphate wasting syndromes. In addition to germ line mesenchymal tumors and solid malignancies, NK T-cell lymphoma can also cause Onc-Ost. Successful antitumor therapy will likely lead to improvement of Onc-Ost.

\section{Statement of Ethics}

Written informed consent was obtained from the patient's next of kin for publication of this case report and accompanying images.

\section{Conflict of Interest Statement}

J.C.Q.V. has participated in consulting for Mallinckrodt Pharmaceuticals and Bayer, advisory board for Mallinckrodt Pharmaceuticals, Travere, and speaker bureau for Otsuka Pharmaceuticals.

\section{Funding Sources}

We declare no funding source for this case report.

\section{Author Contributions}

G.Z., T.N., and J.P.C. were involved in describing the case. S.K. helped drafting Introduction and Discussion. J.C.Q.V. helped with formatting and overall supervision.

\section{References}

1 Feng J, Jiang $\mathrm{Y}$, Wang $\mathrm{O}$, Li M, Xing X, Huo $\mathrm{L}$, et al. The diagnostic dilemma of tumor induced osteomalacia: a retrospective analysis of 144 cases. Endocr J. 2017;64(7):67583.

2 Sauder A, Wiernek S, Dai X, Pereira R, Yudd M, Patel C, et al. FGF23-associated tumor-induced osteomalacia in a patient with small cell carcinoma: a case report and regulatory mechanism study. Int J Surg Pathol. 2016; 24(2):116-20.

3 Lin HA, Shih SR, Tseng YT, Chen $\mathrm{CH}$, Chiu WY, Hsu CY, et al. Ovarian cancer-related hypophosphatemic osteomalacia: a case report. J Clin Endocrinol Metab. 2014;99(12): 4403-7.

4 Bhasin B, Velez JCQ. Persistent urinary phosphate wasting in a patient with metastatic breast cancer: what's your diagnosis? Clin Nephrol. 2021 Feb;95(2):99-103.

5 Elderman JH, Wabbijn M, de Jongh F. Hypophosphataemia due to FGF-23 producing B cell non-Hodgkin's lymphoma. BMJ Case Rep. 2016;2016:bcr2015213954.

6 Hamnvik OP, Becker CB, Levy BD, Loscalzo J. Clinical problem-solving. Wasting away. $\mathrm{N}$ Engl J Med. 2014;370(10):959-66.
7 Mingione A, Maruca K, Chiappori F, Pivari F, Brasacchio C, Quirino T, et al. High parathyroid hormone concentration in tenofovirtreated patients are due to inhibition of calcium-sensing receptor activity. Biomed Pharmacother. 2018;97:969-74.

8 Ramon I, Kleynen P, Body JJ, Karmali R. Fibroblast growth factor 23 and its role in phosphate homeostasis. Eur J Endocrinol. 2010; 162(1):1-10.

9 Liu S, Quarles LD. How fibroblast growth factor 23 works. J Am Soc Nephrol. 2007;18(6): 1637-47.

10 Miyauchi A, Fukase M, Tsutsumi M, Fujita T. Hemangiopericytoma-induced osteomalacia: tumor transplantation in nude mice causes hypophosphatemia and tumor extracts inhibit renal 25-hydroxyvitamin D 1-hydroxylase activity. J Clin Endocrinol Metab. 1988;67(1): 46-53.

11 Jan de Beur SM. Tumor-induced osteomalacia. JAMA. 2005;294(10):1260-7.

12 Huang PY, Li YZ, Yang XM, Yu BQ, Guo L, Guo ZT, et al. [Tumor-induced osteomalacia caused by nasal hemangiopericytoma: a case report]. Zhonghua Er Bi Yan Hou Tou Jing Wai Ke Za Zhi. 2019;54(8):615-7.
13 Tang D, Wang XM, Zhang YS, Mi XX. Oncogenic osteomalacia caused by a phosphaturic mesenchymal tumor of the femur: a case report. World J Clin Cases. 2019;7(15):2081-6.

14 Chong WH, Andreopoulou P, Chen CC, Reynolds J, Guthrie L, Kelly M, et al. Tumor localization and biochemical response to cure in tumor-induced osteomalacia. J Bone Miner Res. 2013;28(6):1386-98.

15 Mitzner SR, Stange J, Klammt S, Risler T, Erley $\mathrm{CM}$, Bader BD, et al. Improvement of hepatorenal syndrome with extracorporeal albumin dialysis MARS: results of a prospective, randomized, controlled clinical trial. Liver Transpl. 2000;6(3):277-86.

16 Rayamajhi SJ, Yeh R, Wong T, Dumeer S, Mittal BR, Remotti F, et al. Tumor-induced osteomalacia: current imaging modalities and a systematic approach for tumor localization. Clin Imaging. 2019;56:114-23.

17 Yeung SJ, McCutcheon IE, Schultz P, Gagel RF. Use of long-term intravenous phosphate infusion in the palliative treatment of tumorinduced osteomalacia. J Clin Endocrinol Metab. 2000;85(2):549-55.

18 Perwad F, Portale AA. Burosumab therapy for $\mathrm{X}$-linked hypophosphatemia and therapeutic implications for CKD. Clin J Am Soc Nephrol. 2019;14(7):1097-9. 